\title{
Attention flexibly trades off across points in time
}

\author{
Rachel N. Denison ${ }^{1} \cdot$ David J. Heeger $^{1} \cdot$ Marisa Carrasco $^{1}$
}

Published online: 4 January 2017

(C) Psychonomic Society, Inc. 2017

\begin{abstract}
Sensory signals continuously enter the brain, raising the question of how perceptual systems handle this constant flow of input. Attention to an anticipated point in time can prioritize visual information at that time. However, how we voluntarily attend across time when there are successive task-relevant stimuli has been barely investigated. We developed a novel experimental protocol that allowed us to assess, for the first time, both the benefits and costs of voluntary temporal attention when perceiving a short sequence of two or three visual targets with predictable timing. We found that when humans directed attention to a cued point in time, their ability to perceive orientation was better at that time but also worse earlier and later. These perceptual tradeoffs across time are analogous to those found across space for spatial attention. We concluded that voluntary attention is limited, and selective, across time.
\end{abstract}

Keywords Temporal attention - Selective attention · Voluntary attention $\cdot$ Visual perception

\section{Introduction}

Visual attention is spatially selective. Covertly attending to a specific location results in perceptual benefits at that location

Electronic supplementary material The online version of this article (doi:10.3758/s13423-016-1216-1) contains supplementary material, which is available to authorized users.

Rachel N. Denison

rachel.denison@nyu.edu

1 Department of Psychology and Center for Neural Science, New York University, New York, NY 10003, USA and costs at unattended ones, compared to when attention is evenly distributed across space (Giordano, McElree, \& Carrasco, 2009; Montagna, Pestilli, \& Carrasco, 2009; Pestilli \& Carrasco, 2005; for reviews, see Carrasco, 2011; Kinchla, 1992). Is visual attention also temporally selective, resulting in perceptual tradeoffs between attended and unattended time points? The answer to this question is unknown, despite its importance to our understanding of dynamic vision.

Previous temporal cueing studies have found that perceptual accuracy and reaction time (RT) are better for attended compared to unattended times (accuracy: Correa, Lupiáñez, \& Tudela, 2005; Davranche, Nazarian, Vidal, \& Coull, 2011; Rohenkohl, Gould, Pessoa, \& Nobre, 2014; Samaha, Bauer, Cimaroli, \& Postle, 2015; RT: Coull \& Nobre, 1998; Miniussi, Wilding, Coull, \& Nobre, 1999; for a review, see Nobre \& Rohenkohl, 2014). But none of these studies were able to assess perceptual tradeoffs between successive targets. The studies that measured accuracy presented only one target per trial and did not compare attended and unattended targets to a neutral baseline, so tradeoffs could not be evaluated. The studies that measured RT suffered from confounds: RT improvements at attended times can arise from motor preparation (Correa, 2010) or criterion changes (Carrasco \& McElree, 2001), along with perceptual improvements.

In attentional blink studies, two targets (usually letters or numbers) are presented in a rapid sequence of nontarget items, and identification of the second target decreases when it appears 200-500 ms after the first (Dux \& Marois, 2009; Raymond, Shapiro, \& Arnell, 1992). Cueing observers to the timing of the second target mitigates this impairment without affecting the first target (Hilkenmeier \& Scharlau, 2010; Martens \& Johnson, 2005; Visser, Tang, Badcock, \& Enns, 2014), suggesting that impairments follow but do not precede an attended item. However, identification accuracy for the first target is typically near ceiling, so cueing during an attentional 
blink task is not ideal to assess tradeoffs mediated by voluntary attention. We developed a new experimental protocol with high sensitivity to perceptual changes to establish the limits of voluntary temporal attention.

We compared four hypotheses. 1) Maximum sustained attention: If observers can sustain attention maximally across time without limits, they have no incentive to use the attentional precue and should always attend to both targets. This hypothesis predicts no difference across attention conditions. One-target cueing studies have generally not found cueing effects for the second of two possible target times, suggesting that observers may be able to sustain attention or rapidly reorient across time (Coull \& Nobre, 1998; Davranche et al., 2011; Rohenkohl et al., 2014). 2) Sustained attention plus enhancement: If observers can sustain attention without limits, but also follow instructions to enhance attention at a particular point in time, they should show attentional benefits but not costs. Previous descriptions of voluntary temporal attention have focused on enhancement (Nobre, 2001). 3) Voluntary attentional blink: If attention decreases following a voluntarily attended target, there should be attentional costs for the second but not the first target. 4) Perceptual tradeoffs: If voluntary temporal attention governs perceptual tradeoffs between the targets, attentional benefits for one target should be mirrored by attentional costs for the other.

\section{Experiment 1}

\section{Methods}

\section{Observers}

Ten observers participated in Experiment 1 (aged 19-43 years, 8 females). Author R.N.D. participated in all three experiments, and four other observers participated in both Experiments 1 and 3. The sample sizes in all experiments were similar to a previous temporal attention study that measured accuracy (Davranche et al., 2011) and to several studies on spatial attention (e.g., Anton-Erxleben, Herrmann, \& Carrasco, 2013). All observers provided informed consent, and the University Committee on Activities involving Human Subjects at New York University approved the experimental protocols. All observers had normal or corrected-to-normal vision.

\section{Stimuli}

Stimuli were generated on an Apple iMac using Matlab and Psychophysics Toolbox (Brainard, 1997; Pelli, 1997; Kleiner, Brainard, \& Pelli, 2007) and were displayed on a gammacorrected Sony Trinitron G520 CRT monitor with a refresh rate of $100 \mathrm{~Hz}$ at a viewing distance of $56 \mathrm{~cm}$. Observers' heads were stabilized by a chin rest. A central white fixation " $\mathrm{x}$ " subtended $0.5^{\circ}$ visual angle. Visual target stimuli were $4 \mathrm{cpd}$ sinusoidal gratings with a 2D Gaussian spatial envelope (standard deviation $0.7^{\circ}$ ), presented in the lower right quadrant of the display centered at $5.7^{\circ}$ eccentricity (Fig. 1a). Stimulus contrast was $64 \%$ and $100 \%$, because we wanted highly visible targets with a short presentation duration. Placeholders, corners of a $4.25^{\circ} \times 4.25^{\circ}$ white square outline (width $0.08^{\circ}$ ) centered on the target location, were present throughout the display to minimize spatial uncertainty. The stimuli were presented on a medium gray background $\left(57 \mathrm{~cd} / \mathrm{m}^{2}\right)$. Auditory cues were high $(784 \mathrm{~Hz}$; G5) or low $(523 \mathrm{~Hz}$; C5) pure sine wave tones, or their combination. Auditory stimuli were presented on the computer speakers at a comfortable volume.

\section{Procedure}

Observers discriminated the orientation of grating patches (Fig. 1a). On each trial, two targets (T1 and T2) were presented at the same spatial location, separated by an interval of 250 ms, an optimal interval for observing the attentional blink (Dux \& Marois, 2009) (Fig. 1b). Each stimulus was tilted slightly clockwise $(\mathrm{CW})$ or counterclockwise $(\mathrm{CCW})$ from either the vertical or horizontal axis, with independent tilts and axes for T1 and T2. Both vertical and horizontal axes were used to reduce the number of trials on which the two stimuli were identical and discourage observers from adopting a strategy of judging whether the stimuli were the same or different to aid in discrimination. An auditory precue 1,000 ms before the first target instructed observers to attend to the first or the second target in selective trials (high tone: $\mathrm{T} 1$; low tone: $\mathrm{T} 2$ ) or to sustain attention across both targets in neutral trials (both tones simultaneously). Observers were asked to report the tilt of one of the two targets. An auditory response cue $500 \mathrm{~ms}$ after T2 indicated which of the two targets to report (high tone: T1; low tone: T2). The timing of auditory and visual events was the same on every trial. From trial to trial, the allocation of temporal attention varied (depending on the precue), and the response selection varied (depending on the response cue). Observers pressed one of two keys to indicate whether the tilt was $\mathrm{CW}$ or $\mathrm{CCW}$ relative to the main axis, with unlimited time to respond. For selective trials ( $80 \%$ of all trials), the response cue matched the precued target with a probability of $75 \%$ (valid trials) and the other target with a probability of $25 \%$ (invalid trials). For neutral trials (20\% of all trials), the two targets were indicated by the response cue with equal probability. Observers received feedback at fixation (correct: green "+"; incorrect: red "-") after each trial, as well as feedback about performance accuracy (percent correct) following each block of trials.

Observers completed two one-hour sessions on separate days. Each session consisted of 5 blocks of 64 trials with all 
a
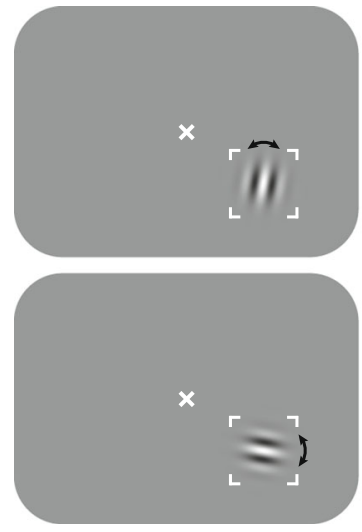

C

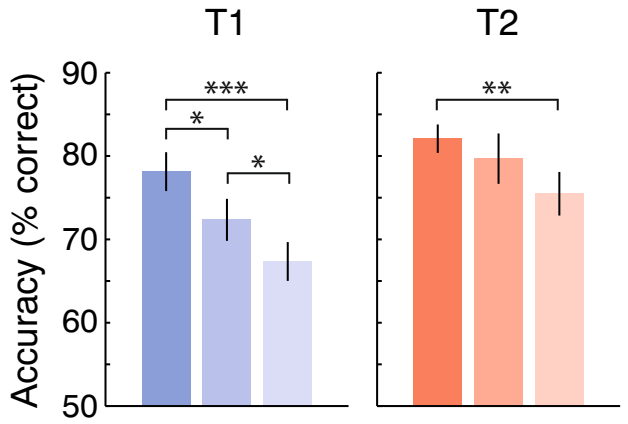

e

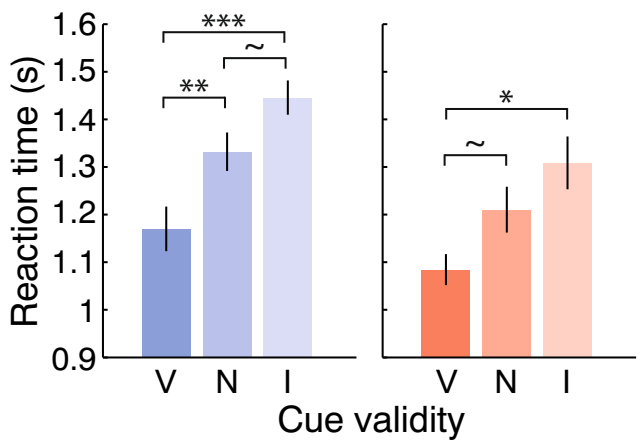

b

T2/both

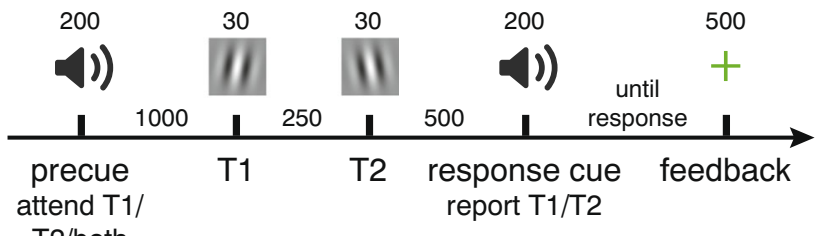

d

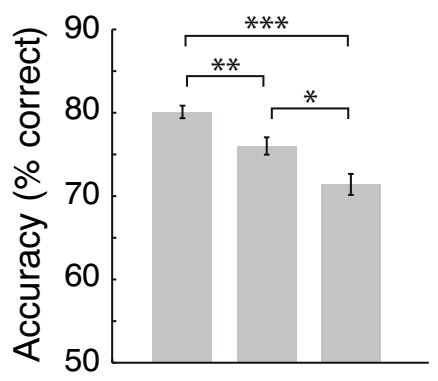

$\mathbf{f}$

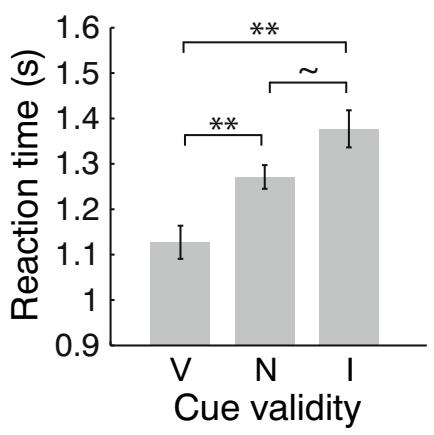

\section{g}

T1 cued T2 cued

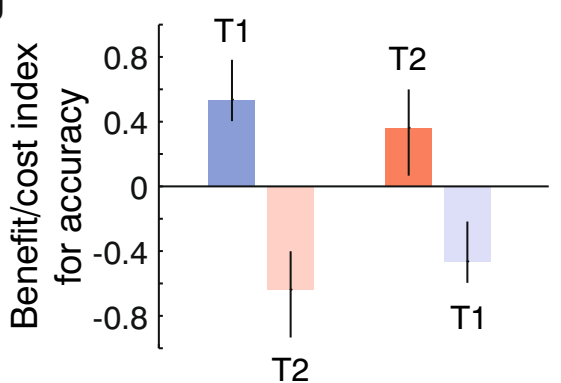

Fig. 1 Experiment 1: Discrimination task and results. (a) Schematic of visual display. Observers judged the tilt (clockwise or counterclockwise) of a sinusoidal grating patch with respect to either the vertical (top) or horizontal (bottom) axis. All stimulus and cueing conditions were presented in randomly interleaved trials. Tilt magnitudes were determined for each observer using a staircase procedure. (b) Trial timeline showing stimulus durations and SOAs in ms. Precues and response cues were pure tones $($ high $=\mathrm{T} 1$, low $=\mathrm{T} 2$, both simultaneously $=$ neutral $)$. $(\mathbf{c})$ Mean accuracy for each temporal cueing condition, pooled across contrast, (d) mean accuracy across targets, (e) mean reaction time for each temporal cueing condition, pooled across contrast, and (f) mean reaction time across targets. Error bars are within-observers SEM (Morey, 2008). V = valid, $\mathrm{N}=$ neutral, $\mathrm{I}=$ invalid. (g) Benefit indices and cost indices (plotted as negative) for accuracy paired according to the cued target. Error bars are $68 \%$ bootstrapped confidence intervals. $\mathrm{n}=10$ observers. $\sim p<0.1$; * $p<0.05 ; * * p<0.01 ; * * * p<0.001$. 
combinations of cue type (valid: $60 \%$, invalid: $20 \%$, neutral: $20 \%)$, stimulus contrast $(64 \%, 100 \%)$, probed target (T1, T2), target tilt (CW, CCW; independent for $\mathrm{T} 1$ and $\mathrm{T} 2)$, and target axis (horizontal, vertical; independent for T1 and T2) in a randomly shuffled order, for a total of 640 trials across the two sessions. Observers completed 64 practice trials at the start of each session.

\section{Training}

Observers also completed one session of training prior to the experiment to familiarize them with the task and determine their tilt thresholds. The training procedure was as follows. First, observers practiced single blocks ( 32 trials each) of the task with neutral precues only, a tilt of 5 degrees (which is typically easy to discriminate), and $80 \%$ contrast stimuli. They repeated this practice condition until they reached $75 \%$ accuracy within a block. Second, they performed 64 trials of the task with a 3-up-1-down staircase, all neutral precues, and $80 \%$ contrast stimuli to determine their individual tilt thresholds. These tilt values were used for the remainder of the experiment. Third, observers completed 64 trials of training with $80 \%$ contrast and all valid precues. Finally, observers completed 320 trials identical to an experimental session. One observer had difficulty discriminating the high and low tones, so tones of $1046.5 \mathrm{~Hz}$ (C6) and $440 \mathrm{~Hz}$ (A4) were used, and extra training was provided.

\section{Eye tracking}

Online eye tracking was used to ensure central fixation throughout the experiment. Initiation of each trial was contingent on fixation, with a 750-ms minimum intertrial interval. Observers were required to maintain fixation, without blinking, from the onset of the precue until the onset of the response cue. If observers broke fixation during this period, the trial was stopped and repeated at the end of the run.

\section{Data analysis}

For each target, we calculated a benefit index and a cost index quantifying the proportion of the total cueing effect that was benefit or cost, according to the following formulae:

benefit index $=\frac{\text { valid-neutral }}{\text { valid-invalid }}$, costindex $=\frac{\text { neutral-invalid }}{\text { valid-invalid }}$.

For each target and cue validity combination (T1 valid, T1 neutral, etc.), we obtained the mean accuracy (Experiments 1 and 2) or precision (Experiment 3 ) across observers and used these values to calculate the benefit and cost indices. The indices generalize across different performance measures, allowing comparisons across experiments, and normalize across targets, which may have different overall cueing effect magnitudes. To assess how benefits for a cued target were related to costs for the other target(s), we paired up the benefit and cost indices in each experiment according to the tradeoffs prediction: the benefit for target A was paired with the cost for target B when A was cued.

\section{Statistics}

Statistical procedures are fully described in Supplementary Information.

\section{Results and discussion}

The results of Experiment 1 provided initial support for the perceptual tradeoffs hypothesis. Accuracy was highest for valid trials (mean across targets $=80 \%$ ), intermediate for neutral trials $(76 \%)$, and lowest for invalid trials $(71 \%)$, reflecting both attentional benefits and costs compared to the neutral condition (Fig. 1c, d; ANOVA main effect of validity: $\left.\mathrm{F}(2,18)=17.64, p<0.0001, \eta_{G}{ }^{2}=0.12\right)$. Performance was comparable for T1 and T2 (no main effect of target or interaction between target and validity, $p>0.1$ ), and all pairwise comparisons among valid, neutral, and invalid trials were significant when combining across targets (valid vs. invalid: $\mathrm{t}(9)$ $=5.96, p=0.0002, \mathrm{~d}=1.89$; valid vs. neutral: $\mathrm{t}(9)=3.84, p=$ $0.004, \mathrm{~d}=1.21$; neutral vs. invalid: $\mathrm{t}(9)=2.58, p=0.03, \mathrm{~d}=$ 0.82 ). Accuracy also was higher on valid compared with invalid trials for each target individually (T1: $\mathrm{t}(9)=7.84, p<$ $0.0001, \mathrm{~d}=2.48 ; \mathrm{T} 2: \mathrm{t}(9)=3.9, p=0.004, \mathrm{~d}=1.23)$. T1 showed significant benefits $(\mathrm{t}(9)=3.14, p=0.012, \mathrm{~d}=0.99$ ) and costs $(\mathrm{t}(9)=2.61, p=0.028, \mathrm{~d}=0.82)$. T2 benefits $(\mathrm{t}(9)=$ $1.48, p=0.17, \mathrm{~d}=0.47)$ and costs $(\mathrm{t}(9)=1.54, p=0.16, \mathrm{~d}=$ $0.48)$ were smaller and not significant. RT showed the same pattern as accuracy, with fastest responses for valid trials, intermediate for neutral, and slowest for invalid trials (Fig. 1e, f; Supplementary Information). These data also establish the temporal width of the voluntary attentional selection window to be shorter than $250 \mathrm{~ms}$; if voluntary attention had less temporal precision, there would be no differences among cueing conditions.

The perceptual tradeoffs hypothesis predicts that cueing one target will benefit that target at the cost of the other target. These benefits and costs could be symmetrical in time (equal magnitude benefits and costs for both targets) or asymmetrical (e.g., strong benefits for T1 and strong costs for T2, but weaker benefits for $\mathrm{T} 2$ and costs for $\mathrm{T} 1$ ). To examine the pattern of benefits and costs and facilitate comparisons across experiments, we calculated for each target a benefit index and a cost index and paired them according to the cued target (see Methods). The similar magnitudes of benefits and costs for each cued target support the tradeoffs hypothesis (Fig. 1g). 


\section{Experiment 2}

Experiment 1 showed clear attentional costs for $\mathrm{T} 1-\mathrm{a}$ decrement in performance before an attended time-contrary to the voluntary attentional blink hypothesis. But the comparison between neutral and invalid was not statistically significant for $\mathrm{T} 2$, so whether attentional costs also occur after an attended time was not clear. We reasoned that precise temporal allocation of attention may be less critical for the last target, because there is extra time to process it. To test whether costs would occur after an attended time if the unattended item was not the last in the sequence, we conducted Experiment 2, with three targets (T1, T2, and T3).

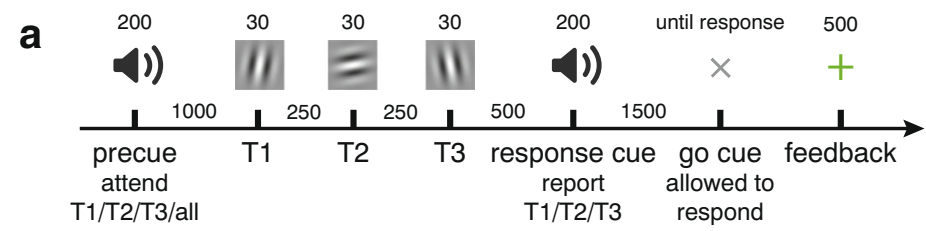

$\mathrm{T} 1$
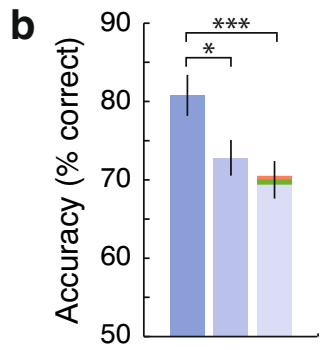

d

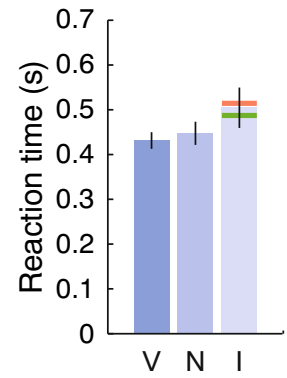

T2
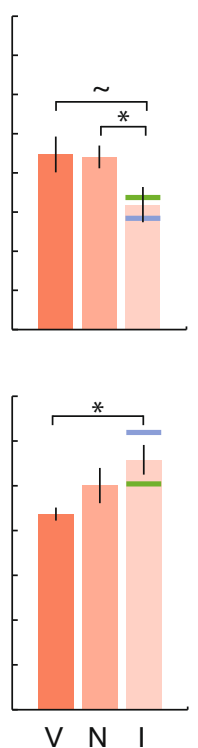

Cue validity

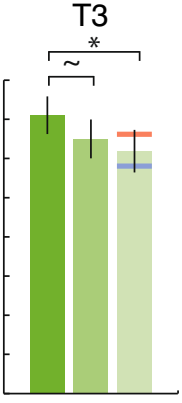

- T1 cued

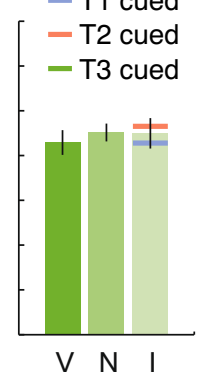

e
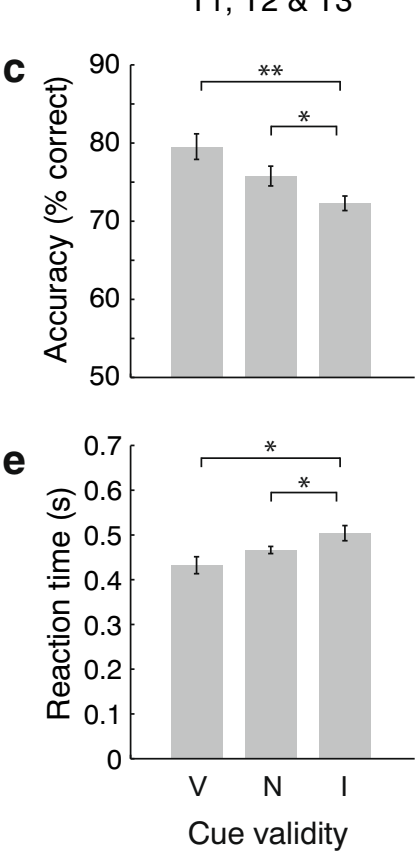

$T 1, T 2 \& T 3$

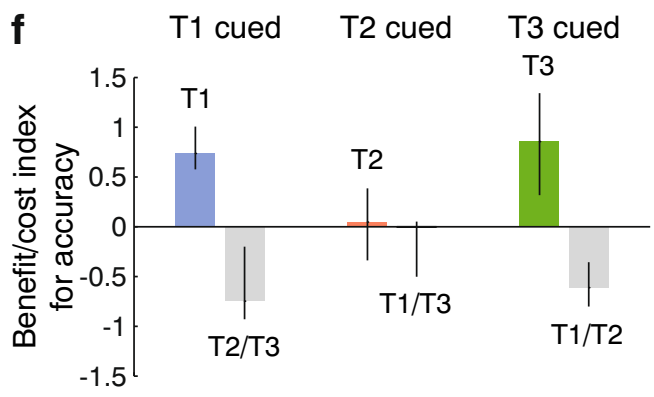

temporal cueing conditions ( $\mathrm{V}=$ valid, $\mathrm{N}=$ neutral, $\mathrm{I}=$ invalid). Colored lines on invalid bars indicate performance conditioned on cued target. (f) Benefit indices and cost indices (plotted as negative) for accuracy calculated separately for each possible target pairing (T1-T2, T1-T3, T2-T3), with costs conditioned on the cued target (colored lines, b). For visualization, the indices were averaged across the two pairings associated with each cued target. Error bars are $68 \%$ bootstrapped confidence intervals. $\mathrm{n}$ $=10$ observers. $\sim p<0.1$; ${ }^{*} p<0.05$; ** $p<0.01$; *** $p<0.001$. 


\section{Methods}

\section{Observers, stimuli, and procedure}

Ten observers participated (19-32 years, 6 females). The task (Fig. 2a) was very similar to the task in Experiment 1, with the following differences. First, there were three targets, with corresponding high (T1: $1318 \mathrm{~Hz}$; E6), medium (T2: $784 \mathrm{~Hz}$; G5), and low (T3: $330 \mathrm{~Hz}$; E4) cue tones. The neutral cue was a combination of the three tones. Second, all stimuli were $100 \%$ contrast. Third, a 1,500-ms delay was enforced before observers were allowed to respond to minimize speedaccuracy tradeoffs. The fixation cross dimmed (the go cue) to signal the beginning of the response period. Therefore, RTs, which were measured with respect to the go cue, were expected to be faster than in Experiment 1, in which there was no forced response delay and RTs were measured with respect to the response cue. Observers were required to maintain fixation (again, monitored by the eye tracker) from the onset of the precue until the onset of the go cue. Observers completed one training session (same structure as Experiment 1) and three one-hour sessions (960 trials) of the experiment.

\section{Results and discussion}

Voluntary temporal attention affected performance accuracy for all three targets, demonstrating its flexibility over subsecond timescales (Fig. 2b, c). Accuracy was again highest for valid $(80 \%)$, intermediate for neutral $(76 \%)$, and lowest for invalid trials $(72 \%)$, with a main effect of cue validity $\left(\mathrm{F}(2,18)=7.68, p=0.0039, \eta_{G}{ }^{2}=0.12\right)$. RTs showed that the changes in accuracy were not due to speed-accuracy tradeoffs (Fig. 2d, e; Supplementary Information). Addressing the question of whether costs in accuracy can occur after an attended time, T2 showed attentional costs overall $(\mathrm{t}(9)=2.94, p=0.017, \mathrm{~d}=0.93)$ and specifically on those trials in which $\mathrm{T} 1$ was cued $(\mathrm{t}(9)=2.80, p=0.021, \mathrm{~d}=0.88)$. These costs were mirrored by attentional benefits for $\mathrm{T} 1 \mathrm{t}(\mathrm{t})$ $=2.28, p=0.049, \mathrm{~d}=0.72)$. T3 had higher accuracy on valid than invalid trials $(\mathrm{t}(9)=2.673, p=0.026, \mathrm{~d}=0.84)$, but did not show reliable benefits and costs. Benefits and costs were less symmetrical for each target compared to Experiment 1 (interaction between cue validity and target, $\mathrm{F}(2,18)=2.81$, $p=0.040, \eta_{G}^{2}=0.033$ ). Thus, there were no costs for $\mathrm{T} 1$ in this experiment, but there were marginal costs for $\mathrm{T} 2$ when $\mathrm{T} 3$ was cued $(\mathrm{t}(9)=2.25, p=0.051, \mathrm{~d}=0.71)$, providing additional support for costs before an attended time. Pairing benefits with their associated costs shows that cueing T1 and T3 resulted in both benefits to those targets and costs to the other targets, but there was no evidence for benefits or costs when T2 was cued (Fig. 2f). The difference between valid and invalid performance accuracy for $\mathrm{T} 2$ was still about half that for $\mathrm{T} 1$ and similar to the $\mathrm{T} 3$ difference. The smaller effects of attentional cueing for $\mathrm{T} 2$ compared with $\mathrm{T} 1$ may have therefore resulted not from extra processing time but from T1 partially disrupting voluntary temporal attention to subsequent targets.

\section{Experiment 3}

In what way does voluntary temporal attention change perception? One possibility is that voluntary temporal attention increases the quality of a visual representation, similar to rhythmic expectation (Cravo, Rohenkohl, Wyart, \& Nobre, 2013; Rohenkohl, Cravo, Wyart, \& Nobre, 2012). Another possibility is that attending to one target results in completely missing another target, similar to the attentional blink (Asplund, Fougnie, Zughni, Martin, \& Marois, 2014; Sergent \& Dehaene, 2004). The discrimination task, with its binary measure of accuracy, could not distinguish between these possibilities. In addition, a trivial explanation for performance tradeoffs would be that on invalid trials, observers mistakenly reported the tilt of the precued target instead of the one indicated by the response cue (as these tilts differed in half of the trials). This possibility must be ruled out to conclude that the tradeoffs are perceptual. To distinguish among these possibilities, we performed an experiment with a graded measure of performance accuracy on each trial.

\section{Methods}

\section{Observers, stimuli, and procedure}

Twelve observers participated (aged 19-43 years, 7 females; 5 also participated in Experiment 1). The procedure was very similar to Experiment 1, with the following differences. First, target orientations were selected randomly and uniformly from $0-180^{\circ}$, with independent orientations for T1 and T2 (Fig. 3a). Second, observers estimated the orientation of the target indicated by the response cue by adjusting a grating patch probe to match the perceived target orientation. The probe was identical to the target but appeared in a new random orientation. Observers moved the mouse horizontally to adjust the orientation of the probe and clicked the mouse to submit the response, with unlimited time to respond. The difference between the reported and presented target orientations was the error for that trial. Third, all stimuli were $100 \%$ contrast. Fourth, observers were given three levels of feedback about the accuracy of their responses on each trial: small error $<5^{\circ}$ (green “+”), medium error $<10^{\circ}$ (yellow “+”), and large error $\geq 10^{\circ}$ (red "-"). Observers were informed about these error ranges. Additional feedback after each block showed the percent of trials with $<5^{\circ}$ errors. 
a

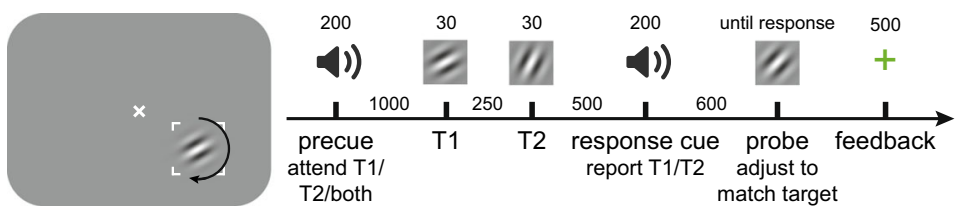

b
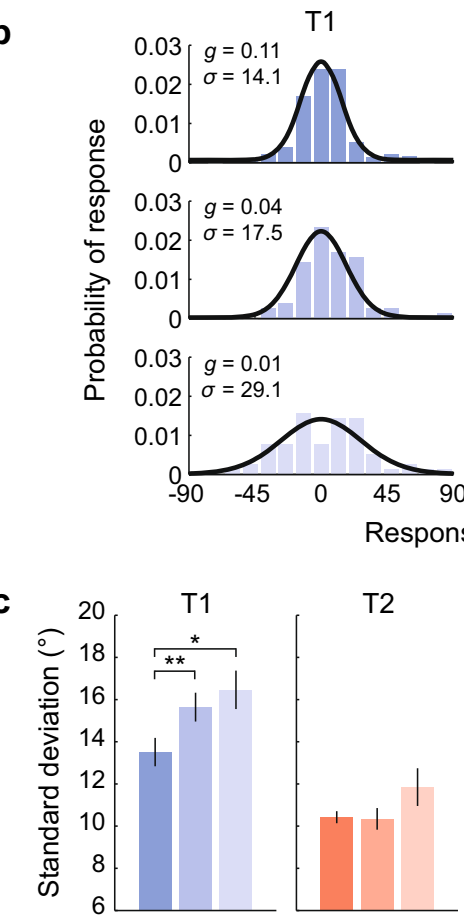

e

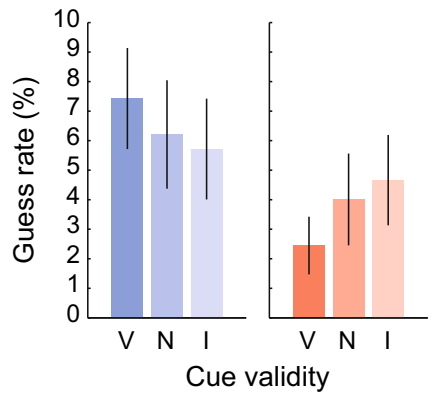

$\mathrm{T} 2$

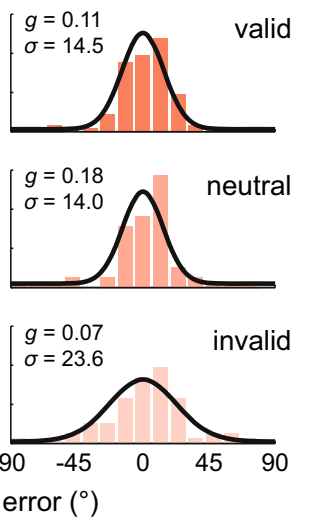

d

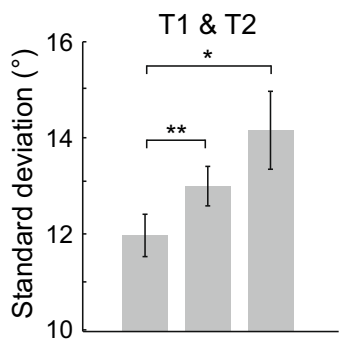

f

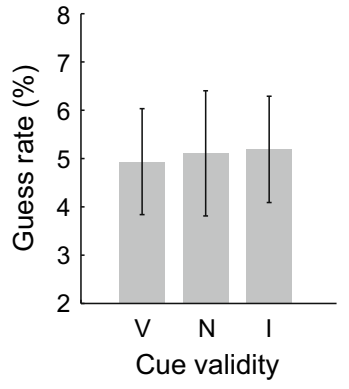

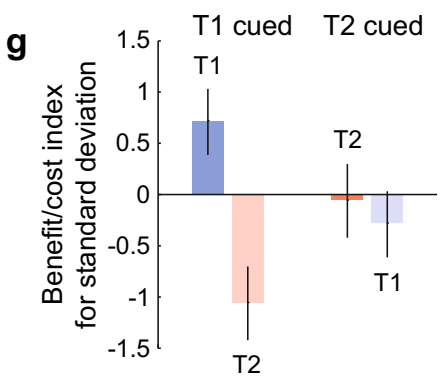

Fig. 3 Experiment 3: Estimation task and results. (a) Schematic of visual display and trial timeline. Targets appeared at random, independent orientations $\left(0-180^{\circ}\right)$, and observers adjusted a probe stimulus to match the perceived orientation of the target indicated by the response cue. (b) Estimation error distributions (colored bars) and model fits (black curves) for an example observer, with the estimated standard deviation $(\sigma)$ and guess rate (g) for each condition. (c) Standard deviation (inverse

precision) parameters estimated from the error distributions for each observer and temporal cueing condition. (d) Standard deviation, mean across targets. (e) Guess rate parameters estimated from the error distributions. (f) Guess rate, mean across targets. (g) Benefit indices and cost indices (plotted as negative) for standard deviation paired according to the cued target. $\mathrm{n}=12$ observers. All other conventions as in Fig. 1. $* p<$ 0.05 ; ** $p<0.01$. 
Each session of the experiment consisted of eight blocks of 40 trials, for a total of 640 trials across two 1-hour sessions. Observers completed 16 practice trials at the start of each session. Observers who had not participated in the discrimination experiment (Experiment 1) completed one session of training before the experiment. The training consisted of practice with all neutral precues (blocks of 16 trials, until reaching criterion of $15 \%$ with $<5^{\circ}$ error), training with all valid precues (64 trials), and training on all precue validities (40 trials).

\section{Analysis}

We modeled the distributions of estimation errors across trials to characterize: 1) the precision of the orientation representation, 2) the rate of all-or-none guessing, 3) the likelihood of target swapping (i.e., reporting the target not indicated by the response cue), and 4) a bias to report clockwise or counterclockwise (Bays, Catalao, \& Husain, 2009; Suchow, Brady, Fougnie, \& Alvarez, 2013; Zhang \& Luck, 2008). Model comparison showed that only the first two parameters, precision and guess rate, were required to capture the data (Supplementary Information). For each experimental condition, we estimated the precision as the standard deviation of a circular normal distribution centered on zero error (inverse precision) and the guess rate as the height of a uniform distribution over orientation (Fig. 3b). Additional modeling details are available in Supplementary Information.

\section{Results and discussion}

Across targets, we found that temporal attention changed the precision of the visual representation (Fig. 3c, d; F $(2,22)=$ 6.41, $p=0.006, \eta_{P}^{2}=0.37$ ) without affecting the guess rate (Fig. 3e, f; $\mathrm{F}(2,22)<1)$. Precision was higher for T2 than T1 $\left(\mathrm{F}(1,11)=57.75, p<0.0001, \eta_{P}{ }^{2}=0.84\right)$, and only $\mathrm{T} 1$ showed reliable precision increases with attention (valid vs. invalid: $\mathrm{Z}=2.20, p=0.027, \mathrm{r}=0.64$; valid vs. neutral: $\mathrm{Z}=2.51$, $p=0.009, \mathrm{r}=0.72)$. Cue validity affected precision across targets even when the swap parameter was included $(\mathrm{F}(2,22)$ $\left.=5.75, p=0.010, \eta_{P}^{2}=0.34\right)$. Thus, changes in visual representations, rather than selection failures or order errors, were responsible for attention-related performance changes. Model comparison rejected the inclusion of a swapping parameter, so there was no evidence that observers mistakenly reported the orientation of the wrong target. The benefit/cost index for precision showed similar-magnitude benefits and costs when T1 was cued, but there was no evidence for benefits or costs when T2 was cued (Fig. 3g).

\section{Performance across experiments}

The perceptual tradeoffs hypothesis predicts that attentional benefits for one target should be mirrored by attentional costs for the other: larger benefits should be accompanied by larger costs, smaller benefits by smaller costs. Overall, this pattern held qualitatively in each experiment, although there was some variability across experiments in the magnitude of benefits and costs across time. We attribute these differences to differences in task demands (see General discussion). We plotted the paired benefit and cost indices across all three experiments together (Fig. 4) and found that the overall pattern of data was consistent with tradeoffs. There was a positive correlation between benefits and their paired costs $(\mathrm{r}=0.80, p=0.0052)$, and the bestfitting regression line fell close to the unity line.

\section{General discussion}

Altogether, these experiments support the perceptual tradeoffs hypothesis. Voluntarily directing attention to a point in time leads to perceptual improvements at that time but also impairments earlier and later. The presence of costs shows that voluntary temporal attention is selective - not only in the sense that it can select a particular moment in time, but in the sense that when it does so, perception at other moments suffers. Such selectivity is the hallmark of voluntary spatial attention (Carrasco, 2011; Giordano et al., 2009; Kinchla, 1992; Montagna et al., 2009; Pestilli \& Carrasco, 2005), but whether

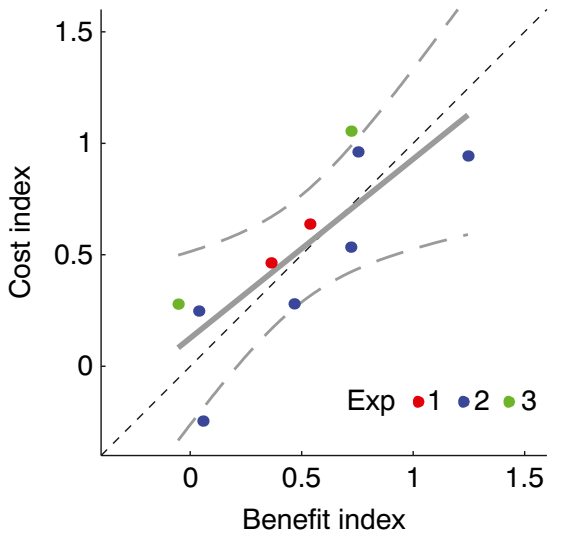

Fig. 4 Experiments 1-3: Perceptual tradeoffs. For each experiment, the attentional benefit index for a given target is plotted against the paired attentional cost index for another target. For example, the benefit for T1 is paired with the cost for T2 when T1 was cued. Red = Experiment 1; blue $=$ Experiment 2; green $=$ Experiment 3 . For Experiment 2 (three targets), benefit and cost indices are calculated and plotted separately for each possible target pairing (T1-T2, T1-T3, T2-T3). The gray solid line shows a linear fit to the data, with $95 \%$ confidence intervals bounded by gray dashed curves. Thin black dashes show the unity line (benefits $=$ costs). 
voluntary attention also is selective in time had not been tested. Selectivity thus is common to both spatial and temporal voluntary attention.

The effects of voluntary temporal attention differ from those seen in the attentional blink. Voluntary temporal attention affects the precision of the visual representation rather than an all-or-none selection process, as has been reported for the blink (Asplund et al., 2014; Goodbourn et al., 2016; Sergent \& Dehaene, 2004), and attentional costs can occur both before and after the attended target. Some experiments have shown that voluntary temporal attention and the attentional blink do not interact, whereas others have found that cued attention benefits the second target more when it is within the attentional blink period (Hilkenmeier \& Scharlau, 2010; Martens \& Johnson, 2005; Visser et al., 2014). It may be that the attentional blink reflects a form of involuntary temporal attentional reduction elicited by the target (Wyble, Potter, Bowman, \& Nieuwenstein, 2011), which is dissociable from voluntary temporal attention, or that it reflects a separate limitation (Chun \& Potter, 1995). Multiple processes are likely involved in performing the attentional blink task (e.g., target selection, distractor suppression, letter/number identification; Dux \& Marois, 2009). In contrast, the perceptual tradeoffs we observed can be unambiguously linked to temporal attention, because attention was explicitly manipulated.

The variability in the specific cueing effects found across experiments may reflect different attentional demands and strategies for different displays and tasks. For example, with two targets (Experiment 1), there were roughly symmetrical benefits and costs for both targets; whereas with three targets (Experiment 2), there were benefits for $\mathrm{T} 1$ and $\mathrm{T} 3$ and corresponding costs for T2, but not vice versa. A possible explanation for this difference is that voluntary temporal attention is most effective when cued to the beginning or end of a sequence. In Experiment 3, precision was much higher for T2 than for T1, perhaps reflecting differences between estimation and discrimination tasks. This may have left little room to measure attentional cueing effects for $\mathrm{T} 2$. The correlation analysis shows that the tradeoffs hypothesis - the correspondence of benefits and costs - provides a parsimonious explanation for the pattern of data across varying experimental conditions. In the future, it will be interesting to study a wider variety of tasks and displays, including longer sequences.

The finding that observers cannot sustain attention over a sequence of just two target stimuli adds to previous findings of temporal processing limitations for longer, more rapid sequences, and sequences with irrelevant (nontarget) items (Broadbent \& Broadbent, 1987; Duncan, Ward, \& Shapiro, 1994; Nieuwenstein, Van der Burg, Theeuwes, Wyble, \& Potter, 2009; Reeves \& Sperling, 1986; Weichselgartner \& Sperling, 1987). It suggests limitations in visual processing over short time intervals $(<1 \mathrm{~s})$, rather than a strategic disruption of attention when an irrelevant item appears (Olivers \&
Meeter, 2008), consistent with accounts of resource sharing over time (Shapiro, Schmitz, Martens, Hommel, \& Schnitzler, 2006; Wieser \& Keil, 2011). Our findings show that voluntary temporal attention is not a process that can be sustained at will in the presence of multiple targets, nor a process that simply boosts sensory information at a relevant time. Rather, it is a flexible, cognitive selection process that serves to manage limits in visual perception across time, perhaps rendering them less noticeable in daily life.

Acknowledgments Funding was provided by National Institutes of Health National Eye Institute grants R01 EY019693 to D.J.H. and M.C., F32 EY025533 to R.N.D, and T32 EY007136 to NYU supporting R.N.D. We thank Katherine Chen and Jacob Parker for assistance with data collection and members of the Carrasco Lab for helpful discussions.

\section{References}

Anton-Erxleben, K., Herrmann, K., \& Carrasco, M. (2013). Independent effects of adaptation and attention on perceived speed. Psychological Science, 24(2), 150-159. doi:10.1177/0956797612449178

Asplund, C. L., Fougnie, D., Zughni, S., Martin, J. W., \& Marois, R. (2014). The attentional blink reveals the probabilistic nature of discrete conscious perception. Psychological Science, 25(3), 824-831. doi: $10.1177 / 0956797613513810$

Bays, P. M., Catalao, R. F. G., \& Husain, M. (2009). The precision of visual working memory is set by allocation of a shared resource. Journal of Vision, 9(10):7, 1-11. doi: 10.1167/9.10.7

Brainard, D. H. (1997). The Psychophysics Toolbox. Spatial Vision, 10(4), 433-436.

Broadbent, D. E., \& Broadbent, M. H. P. (1987). From detection to identification: Response to multiple targets in rapid serial visual presentation. Perception \& Psychophysics, 42(2), 105-113. doi:10.3758/BF03210498

Carrasco, M. (2011). Visual attention: The past 25 years. Vision Research, 51(13), 1484-1525. doi:10.1016/j.visres.2011.04.012

Carrasco, M., \& McElree, B. (2001). Covert attention accelerates the rate of visual information processing. Proceedings of the National Academy of Sciences of the United States of America, 98(9), 5363-5367. doi:10.1073/pnas.081074098

Chun, M. M., \& Potter, M. C. (1995). A two-stage model for multiple target detection in rapid serial visual presentation. Journal of Experimental Psychology: Human Perception and Performance, 21(1), 109-127.

Correa, A. (2010). Enhancing behavioural performance by viual temporal orienting. In A. C. Nobre \& J. T. Coull (Eds.), Attention and Time. Oxford, UK: Oxford University Press.

Correa, A., Lupiáñez, J., \& Tudela, P. (2005). Attentional preparation based on temporal expectancy modulates processing at the perceptual level. Psychonomic Bulletin \& Review, 12(2), 328-334.

Coull, J. T., \& Nobre, A. C. (1998). Where and when to pay attention: The neural systems for directing attention to spatial locations and to time intervals as revealed by both PET and fMRI. Journal of Neuroscience, 18(18), 7426-7435.

Cravo, A. M., Rohenkohl, G., Wyart, V., \& Nobre, A. C. (2013). Temporal expectation enhances contrast sensitivity by phase entrainment of low-frequency oscillations in visual cortex. Journal of Neuroscience, 33(9), 4002-4010. doi:10.1523/JNEUROSCI.467512.2013

Davranche, K., Nazarian, B., Vidal, F., \& Coull, J. (2011). Orienting attention in time activates left intraparietal sulcus for both perceptual 
and motor task goals. Journal of Cognitive Neuroscience, 23(11), 3318-3330. doi:10.1162/jocn_a 00030

Duncan, J., Ward, R., \& Shapiro, K. (1994). Direct measurement of attentional dwell time in human vision. Nature, 369(6478), 313-315. doi:10.1038/369313a 0

Dux, P. E., \& Marois, R. (2009). The attentional blink: A review of data and theory. Attention, Perception, \& Psychophysics, 71(8), 16831700. doi:10.3758/APP.71.8.1683

Giordano, A. M., McElree, B., \& Carrasco, M. (2009). On the automaticity and flexibility of covert attention: A speed-accuracy trade-off analysis. Journal of Vision, 9(3):30, 1-10. doi: 10.1167/9.3.30

Goodbourn, P. T., Martini, P., Barnett-Cowan, M., Harris, I. M., Livesey, E. J., \& Holcombe, A. O. (2016). Reconsidering temporal selection in the attentional blink. Psychological Science, 27(8), 1146-1156. doi: $10.1177 / 0956797616654131$

Hilkenmeier, F., \& Scharlau, I. (2010). Rapid allocation of temporal attention in the attentional blink paradigm. European Journal of Cognitive Psychology, 22(8), 1222-1234. doi:10.1080/09541440903418924

Kinchla, R. A. (1992). Attention. Annual Review of Psychology, 43, 711742. doi:10.1146/annurev.ps.43.020192.003431

Kleiner, M., Brainard, D., \& Pelli, D. (2007). What's new in Psychtoolbox-3? Perception 36 ECVP Abstract Supplement

Martens, S., \& Johnson, A. (2005). Timing attention: Cuing target onset interval attenuates the attentional blink. Memory \& Cognition, 33(2), 234-240.

Miniussi, C., Wilding, E. L., Coull, J. T., \& Nobre, A. C. (1999). Orienting attention in time: Modulation of brain potentials. Brain, 122(Pt 8), 1507-1518.

Montagna, B., Pestilli, F., \& Carrasco, M. (2009). Attention trades off spatial acuity. Vision Research, 49(7), 735-745.

Morey, R. D. (2008). Confidence intervals from normalized data: A correction to Cousineau (2005). Tutorial in Quantitative Methods for Psychology, 4(2), 61-64.

Nieuwenstein, M., Van der Burg, E., Theeuwes, J., Wyble, B., \& Potter, M. (2009). Temporal constraints on conscious vision: On the ubiquitous nature of the attentional blink. Journal of Vision, 9(9):18, 114. doi: $10.1167 / 9.9 .18$

Nobre, A. C. (2001). Orienting attention to instants in time. Neuropsychologia, 39(12), 1317-1328.

Nobre, A. C., \& Rohenkohl, G. (2014). Time for the fourth dimension in attention. In A. C. Nobre \& S. Kastner (Eds.), The Oxford Handbook of Attention. Oxford, UK: Oxford University Press.

Olivers, C. N. L., \& Meeter, M. (2008). A boost and bounce theory of temporal attention. Psychological Review, 115(4), 836-863. doi:10.1037/a0013395

Pelli, D. G. (1997). The VideoToolbox software for visual psychophysics: Transforming numbers into movies. Spatial Vision, 10(4), 437-442.
Pestilli, F., \& Carrasco, M. (2005). Attention enhances contrast sensitivity at cued and impairs it at uncued locations. Vision Research, 45(14), 1867-1875. doi:10.1016/j.visres.2005.01.019

Raymond, J. E., Shapiro, K. L., \& Arnell, K. M. (1992). Temporary suppression of visual processing in an RSVP task: An attentional blink? Journal of Experimental Psychology: Human Perception and Performance, 18(3), 849-860.

Reeves, A., \& Sperling, G. (1986). Attention gating in short-term visual memory. Psychological Review, 93(2), 180-206.

Rohenkohl, G., Cravo, A. M., Wyart, V., \& Nobre, A. C. (2012). Temporal expectation improves the quality of sensory information. Journal of Neuroscience, 32(24), 8424-8428. doi:10.1523/JNEUROSCI.080412.2012

Rohenkohl, G., Gould, I. C., Pessoa, J., \& Nobre, A. C. (2014). Combining spatial and temporal expectations to improve visual perception. Journal of Vision, 14(4):8, 1-13. doi: 10.1167/14.4.8

Samaha, J., Bauer, P., Cimaroli, S., \& Postle, B. R. (2015). Top-down control of the phase of alpha-band oscillations as a mechanism for temporal prediction. Proceedings of the National Academy of Sciences of the United States of America. doi:10.1073/pnas.1503686112

Sergent, C., \& Dehaene, S. (2004). Is consciousness a gradual phenomenon? Evidence for an all-or-none bifurcation during the attentional blink. Psychological Science, 15(11), 720-728. doi:10.1111/j.09567976.2004.00748.x

Shapiro, K., Schmitz, F., Martens, S., Hommel, B., \& Schnitzler, A. (2006). Resource sharing in the attentional blink. Neuroreport, 17(2), 163-166.

Suchow, J. W., Brady, T. F., Fougnie, D., \& Alvarez, G. A. (2013). Modeling visual working memory with the MemToolbox. Journal of Vision, 13(10):9, 1-8. doi: 10.1167/13.10.9.doi

Visser, T. A. W., Tang, M. F., Badcock, D. R., \& Enns, J. T. (2014). Temporal cues and the attentional blink: A further examination of the role of expectancy in sequential object perception. Attention, Perception, \& Psychophysics, 76(8), 2212-2220. doi:10.3758/s13414-014-0710-7

Weichselgartner, E., \& Sperling, G. (1987). Dynamics of automatic and controlled visual attention. Science, 238(4828), 778-780.

Wieser, M. J., \& Keil, A. (2011). Temporal trade-off effects in sustained attention: Dynamics in visual cortex predict the target detection performance during distraction. Journal of Neuroscience, 31(21), 7784-7790. doi:10.1523/JNEUROSCI.5632-10.2011

Wyble, B., Potter, M. C., Bowman, H., \& Nieuwenstein, M. (2011). Attentional episodes in visual perception. Journal of Experimental Psychology: General, 140(3), 488-505. doi:10.1037/a0023612

Zhang, W., \& Luck, S. J. (2008). Discrete fixed-resolution representations in visual working memory. Nature, 453(7192), 233-235. doi: 10.1038 /nature 06860 